\title{
Normal Insulin Secretion from Immune-Protected Islets of Langerhans by PEGylation and Encapsulation in the Alginate-Chitosan-PEG
}

\author{
Nahid Najafikhah ${ }^{1}$, Sameereh Hashemi-Najafabadi ${ }^{1 *}$, Saleh Zahediasl ${ }^{2}$, Mohammad Mahdi Nabavimanesh \\ ${ }^{1}$, Khadijeh Farrokhfall ${ }^{3}$ \\ ${ }^{1}$ Biomedical Engineering Department, Faculty of Chemical Engineering, Tarbiat Modares University, Tehran, Iran \\ ${ }^{2}$ Endocrine Research Center, Research Institute of Endocrine Sciences, Shaheed Beheshti University of Medical Sciences, Tehran, Iran \\ ${ }^{3}$ Medical Toxicology and Drug Abuse Research Center (MTDR C), Birjand University of Medical Sciences, Birjand, Iran \\ "Corresponding author: Sameereh Hashemi-Najafabadi, Biomedical Engineering Department, Faculty of Chemical Engineering, Tarbiat Modares University, \\ Tehran, Iran. Tel: +98-2182884384, Fax: +98-2182884931, E-mail: s.hashemi@modares.ac.ir
}

Received: 17 Jul. 2016; $\quad$ Revised: 4 May.2018; $\quad$ Accepted: 17 Jun. 2018； Published online: 12. Dec. 2018

\begin{abstract}
Background: Pancreatic islet transplantation is one of the most promising strategies for treating patients with type I diabetes mellitus.

Objective: We aimed to assess the immunoisolation properties of the multilayer encapsulated islets using alginate-chitosanPEG for immunoprotection and insulin secretion from the encapsulated islets induced under different glucose concentrations in vitro.

Materials and Methods: In this study, the islets were isolated from Wistar rats. The biological function (insulin secretion) of the immunoisolated islets following to PEGylation and encapsulation in the alginate-chitosan-PEG, separately, in addition to their immuno-protection in a co-culturing with the lymphocytes isolated from the male C57BL/6 mice were investigated, respectively.

Results: Alginate-chitosan-PEG decreased IL-2 secretion from the lymphocytes co-cultured with islets. Also, insulin secretion from the encapsulated and PEGylated groups was stimulated by glucose (i.e., 5.6 and $16.7 \mathrm{mM}$ of glucose, respectively); showed insulin secretion similar to the naked islets, without coating, after 30 and 60 min of incubation.

Conclusion: In conclusion, encapsulation and PEGylation have no negative effect on the insulin secretion and glucose sensitivity of the islets for all of the groups. Also, encapsulation decreased IL-2 secretion from the lymphocytes.

Keywords: Alginate; Chitosan; Islets of Langerhans; Insulin ;Type I diabetes mellitus
\end{abstract}

\section{Background}

Type I diabetes mellitus (TIDM) is an autoimmune disease that involves the immunological destruction of the insulin producing islets of Langerhans. Pancreatic islet transplantation is one of the most promising strategies for these patients $(1,2)$. However, this strategy is limited due to the side effects of the immune suppressive drugs that should be taken by the recipients to overcome the host immunological responses, especially in xenotransplantation $(3,4)$. To avoid the host-rejection, in addition to decreasing the risk of immunosuppressive drugs, immuno-isolation systems including microencapsulation for the islets of Langerhans (5-9) and surface modification by polyethylene glycol (PEG) (PEGylation) for pancreatic islets (10-12), have been developed.

Several reactive derivatives of methoxy polyethylene glycol (mPEG) have been used for cell PEGylation. In our previous work, red blood cells (RBCs) were PEGylated by mPEG-succinimdyl valerate (mPEG-

Copyright (C) 2018 The Author(s); Published by National Institute of Genetic Engineering and Biotechnology. This is an open access article, distributed under the terms of the Creative Commons Attribution-NonCommercial 4.0 International License (http://creativecommons.org/licenses/ by-nc/4.0/) which permits others to copy and redistribute material just in noncommercial usages, provided the original work is properly cited. 
SVA) and mPEG-succinimidyl carbonate (mPEG-SC), separately. The results showed that mPEG-SVA was more effective (13).

Additionally, Aghajani-Lazarjani et al. optimized islets PEGylation using mPEG-SPA. The factors of reaction time, the ratio of longer polymer in the mixture of mPEGs, and polymer concentration were considered (14). In another study involving islet PEGylation using mPEG-succinimdyl propionic acid (mPEGSPA) and mPEG-SC, separately, it was shown a better immunological protection using mPEG-SC (10). As well, in our previous study (the data not shown) the results showed that mPEG-SVA had more efficiency than mPEG-SC to protect the islets from the immune system.

Pancreatic islets may also be encapsulated in the semi-permeable membranes. The membranes are designed to allow diffusion of the glucose and insulin but prevent immunological intermediates (5).

Several studies have so far been presented on the properties of the different materials for encapsulation such as chitosan, which is a natural polymer obtained from chitin after $\mathrm{N}$-deacetylation by alkaline treatment. Chitosan is non toxic, biocompatible, and has antimicrobial properties (15). Alginate-poly-L-lysine (PLL) capsules for pancreatic islets were introduced by Lim and Sun (8). In addition, many researchers have investigated encapsulation using different polycations such as chitosan and poly-L-ornithine (PLO). Azadi et al, have used dextran-spermine as a polycation together with alginate as a polyanion for coencapsulation of pancreatic islets and pentoxifylline as an immunosuppressive drug to develop a cell therapy system for the possible treatment of insulin dependent diabetes mellitus (16).

Encapsulation containing three layers is a more protective immunoisolation system. However, these three layers increase the total volume of the microcapsules. In our previous study (17), we introduced a novel immunoisolation system with three layers, using a combination of encapsulation and PEGylation containing alginate-PLO-mPEG that reduces the microcapsules' volume in comparison to the previous three layer encapsulations. Figure 1 shows a schematic picture of the islet encapsulation, PEGylation and also, a combination of PEGylation and encapsulation.

\section{Objectives}

In the present study, the immunoisolation properties of the multilayer encapsulated islets using alginatechitosan-PEG was investigated for immunoprotection and insulin secretion from the encapsulated islets induced under different glucose concentrations.

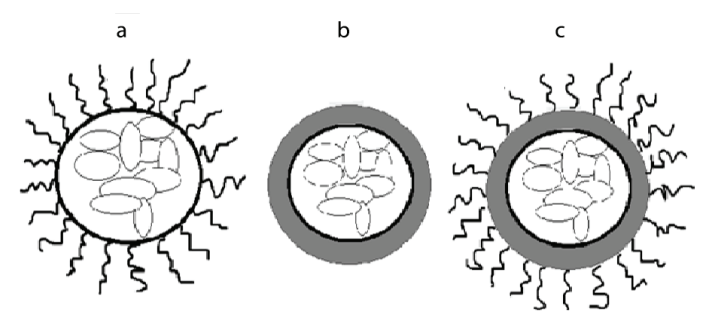

Figure 1. A schematic drawing of (a) PEGylation, (b) encapsulation, and (c) a combination of encapsulation and PEGylation.

\section{Materials and Methods}

\subsection{Materials}

mPEG-SVA of 5 and $10 \mathrm{kDa}$ were purchased from Lysan Bio Inc. Chitosan ( $85 \%$ deacetylation), propidium iodide (PI) and HEPES were obtained from Sigma Aldrich. RPMI-1640 medium, fetal bovine serum (FBS), nonessential amino acid solution (MEM), and penicillin/ streptomycin antibiotics were purchased from GIBCO. Alginate with high $\mathrm{G}$ block content was obtained from BDH. Bovine serum albumin (BSA) and acridine orange (AO) were purchased from Merck. Mouse IL-2 kit was obtained from Ebioscience (San Diego, CA, USA). Insulin ultrasensitive kit and collagenase $P$ were purchased from Mercodia and Roche, respectively.

\subsection{Islet Isolation}

Following $16 \mathrm{~h}$ fasting, adult male Wistar rats (170$190 \mathrm{~g}, 2.5-3.0$ months old), were anesthetized and their abdomen was opened. Briefly, according to the previous study in our group $10 \mathrm{~mL}$ of collagenase (0.5 mg. $\left.\mathrm{mL}^{-1}\right)$ in HBSS was gently injected into the common bile duct. The inflated pancreas was incubated in water bath at $37{ }^{\circ} \mathrm{C}$ for $17 \mathrm{~min}$ and then the digestion process was terminated using cold Hanks solution. The tube was shaken for $1 \mathrm{~min}$ and the obtained suspension was filtered (using $200 \mu \mathrm{m}$ filter) into a glass container. The supernatant was removed, a process which was repeated three times. After the last aspiration, the islets were handpicked (all steps were performed on ice) (10).

\subsection{PEGylation of the Islets}

According to our previous work (17), a group of the isolated islets was handpicked and suspended in the Hanks solution (30 islets. $\mathrm{mL}^{-1}$ ) and mixed with a mixture of $5 \mathrm{kDa}$ mPEG-SVA $(25 \%)$ and $10 \mathrm{kDa}$ mPEG-SVA (75\%). Then, they were incubated at 37 ${ }^{\circ} \mathrm{C}$ and $5 \% \mathrm{CO}_{2}$ condition for $60 \mathrm{~min}$. After washing the PEGylated islets, they were investigated for insulin 
secretion according to the following sections (all steps were performed on ice).

\subsection{Encapsulation of the Islets Using Alginate Chitosan-PEG}

A group of the isolated islets were suspended in alginate solution $(2 \% \mathrm{w} / \mathrm{v}$ containing $2 \mathrm{~g}$ alginate in $100 \mathrm{~mL}$ normal saline) (30 islets. $\left.\mathrm{mL}^{-1}\right)$. Then, using a syringe pump and encapsulator device, it was dropped $\left(7 \mathrm{~mL} \cdot \mathrm{min}^{-1}\right)$ into $\mathrm{CaCl}_{2}$ solution $(100$ $\mathrm{mM}$ ) in which the hydrogel was constructed. The obtained microcapsules remained in $\mathrm{CaCl}_{2}$ solution for 15-30 $\mathrm{min}$. The encapsulated islets were then suspended in chitosan solution $(1 \% \mathrm{w} / \mathrm{v}$ containing $0.1 \mathrm{~g}$ chitosan dissolved in $10 \mathrm{~mL}$ acetic acid $0.1 \mathrm{~N}$ ) for $15 \mathrm{~min}$. The microcapsules were then diluted in mPEG-SVA solution $(0.1 \% \mathrm{w} / \mathrm{v})$ in Hanks' balanced salt solution (HBSS, $\mathrm{pH} 8$ ) for $30 \mathrm{~min}$ (all of the steps were performed on the ice) (18) After washing, the encapsulated islets they were investigated for the insulin secretion and immunological reactions according to the following sections.

\subsection{Leukocytes Isolation}

The leukocytes were isolated from male C57BL/6 mice (12 weeks, 25-30 g), according to the previous work in our group (10).

\subsubsection{Immunological Reactions Investigation}

The immunological reactions were investigated by coculturing 12-16 encapsulated or free islets (without any coating, as control) with $2 \times 10^{5}$ leukocytes, according to our previous work (18).

\subsubsection{Glucose-Stimulated Insulin Secretion}

Glucose-stimulated insulin secretion was assessed at two glucose concentrations ( 5.6 and $16.7 \mathrm{mM}$ ) for 3 groups of the isolated islets (islets without any coating (as control), encapsulated islets, and PEGylated islets), separately. For each glucose concentration, 8 replications of 4 islets were randomly selected and placed in 24-well plates. Two milliliter of Krebs Ringer solution [117 mmoL.L $\mathrm{L}^{-1}$ $\mathrm{NaCl}, 2.5 \mathrm{mmoL} . \mathrm{L}^{-1} \mathrm{CaCl}_{2}, 1.2 \mathrm{mmoL} \cdot \mathrm{L}^{-1} \mathrm{KH}_{2} \mathrm{PO}_{4}$, $1.2 \mathrm{mmoL} . \mathrm{L}^{-1} \mathrm{MgSO}_{4}$, and $24.6 \mathrm{mmoL} . \mathrm{L}^{-1} \mathrm{NaHCO}_{3}$, (pH 7.4), BSA 0.5 g. $\mathrm{dL}^{-1}$ ] containing 5.6 or $16.7 \mathrm{mM}$ glucose was added to each well and incubated at $37^{\circ} \mathrm{C}$ and $5 \% \mathrm{CO}_{2}$ for $60 \mathrm{~min}$. After 30 and $60 \mathrm{~min}, 500 \mu \mathrm{L}$ of the supernatant was sampled from each well and stored at $-70{ }^{\circ} \mathrm{C}$ for insulin analysis $(10,19)$. The amount of insulin secreted by the islets in all groups and under two different glucose concentrations, were measured using rat insulin Elisa kit (19).

\subsection{Viability Test}

Viability of the islets was assessed by microscopic examination using AO-PI staining in each group after $60 \mathrm{~min}$ incubation in glucose solution. The islets were exposed to $50 \mu \mathrm{L} \mathrm{AO}$ and $950 \mu \mathrm{L} \mathrm{PI}$ in $1 \mathrm{~mL}$ incubation solution and evaluated using the fluorescence microscope (Nikon, Japan). The viable and dead cells were identified as green- and redcolored, respectively (10).

\subsection{Statistical Analysis}

Statistical analysis was carried out using PRISM version 5.0 program package with data expressed as mean \pm SEM. Two-way ANOVA followed by Bonferroni test were used to analyze the difference between the groups. P-value less than 0.05 was considered statistically significant.

\section{Results}

\subsection{The Effects of Encapsulation and PEGylation on the Islet Viability}

The isolated islets were PEGylated and encapsulated, in separated groups, according to sections 3.3 and 3.4, and their viability were investigated. As it can be seen in Figure 2, the PEGylated, encapsulated, and control islets, incubated in Krebs Ringer solution ( $\mathrm{pH}$ 7.4) containing 5.6 or $16.7 \mathrm{mM}$ glucose, respectively, were viable after $60 \mathrm{~min}$ incubation.

\subsection{The Effects of Islet Immuno-isolation}

The amounts of IL-2 secreted from the co-cultured
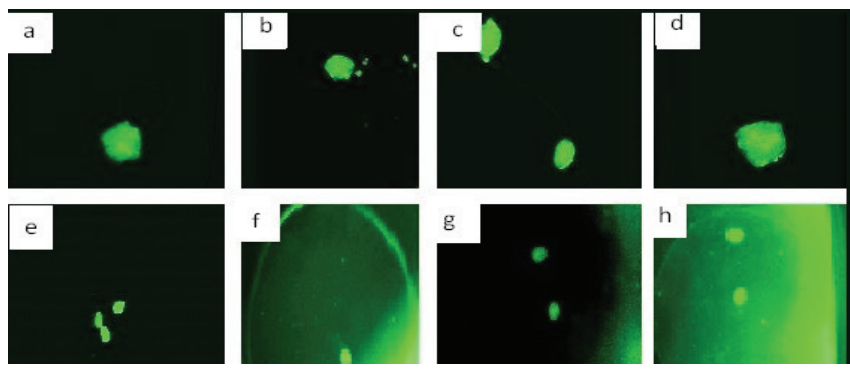

Figure 2. The viability (using AO-PI staining) of the control and PEGylated islets: the viable (a) and control (b) PEGylated islets incubated in the Krebs Ringer solution ( $\mathrm{pH}$ 7.4) containing $5.6 \mathrm{mM}$ glucose after $60 \mathrm{~min}$ incubation. (c) control and (d) PEGylated islets incubated in the Krebs Ringer solution (pH 7.4) containing $16.7 \mathrm{mM}$ glucose after $60 \mathrm{~min}$ incubation. (e) control and (f) encapsulated islets incubated in the Krebs Ringer solution (pH 7.4) containing $5.6 \mathrm{mM}$ glucose after 60 min incubation, and $(\mathrm{g})$ control and $(\mathrm{h})$ the encapsulated islets incubated in the Krebs Ringer solution (pH 7.4) containing $16.7 \mathrm{mM}$ glucose after $60 \mathrm{~min}$ incubation. 


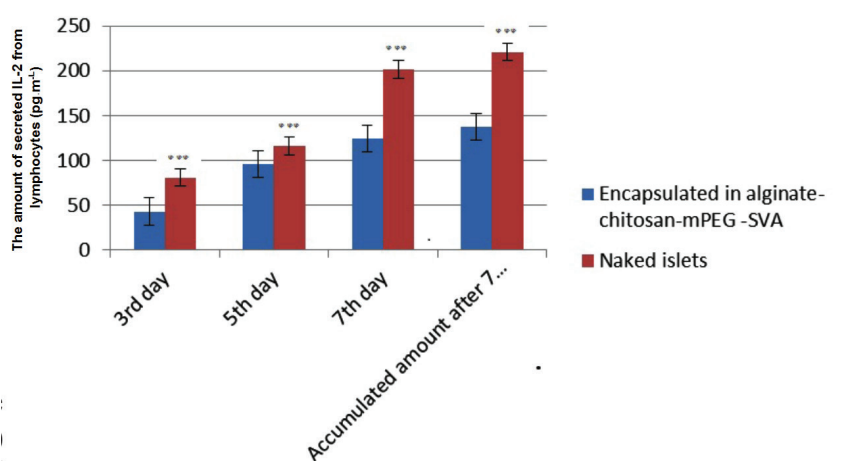

Figure 3. The amount of secreted IL-2 from lymphocytes co-cultured with the islets (*** shows the significant differences).

lymphocytes with the encapsulated islets in alginatechitosan-PEG are presented in Figure 3. According to the results, the amount of IL-2 secretion from the co-cultured lymphocytes with the encapsulated islets after 7 days is approximately 38\% lower than that of co-cultured lymphocytes with the free islets. The immunoprotection of PEGylated islets has also been investigated in another study where it has shown a decrease in the IL-2 secretion in comparison to the free islets, as well (17).

\subsection{The Effect of PEGylation and Encapsulation on Insulin Secretion}

According to section 3.7, glucose-stimulated insulin secretion was assessed at two glucose concentrations (5.6 and $16.7 \mathrm{mM}$ ) for 3 groups of the isolated islets. Insulin secretion from the PEGylated, encapsulated, and control groups are shown in Figure 4. In the presence of $16.7 \mathrm{mM}$ glucose, insulin secretion from the PEGylated and encapsulated groups were significantly $(p<0.001)$ higher than that of $5.6 \mathrm{mM}$ after 30 and $60 \mathrm{~min}$ incubation. Also, the PEGylated and encapsulated groups secreted insulin similar to the control group at 30 and $60 \mathrm{~min}$ (approximately 2.53.0 folds higher for $16.7 \mathrm{mM}$ in all groups). Two-way ANOVA showed no significant differences between insulin secretions from the PEGylated and encapsulated with control islets induced when glucose at 5.6 and $16.7 \mathrm{mM}$ concentrations were used after 30 and $60 \mathrm{~min}$, respectively. The trends in insulin secretion from the PEGylated and encapsulated islets were similar to the control group.

\section{Discussion}

The present study shows the effect of islets encapsulation in the alginate-chitosan-PEG on IL-2 secretion by lymphocytes co-cultured with islets as well as insulin secretion by the encapsulated islets. In the previous studies, Aghajani-Lazarjani et al. (10) have reported the efficiency of mPEG-SPA and mPEG$\mathrm{SC}$ as immunoisolation systems and showed that both activated mPEGs can protect islets from the host immune system. They showed that mPEG-SC is more desirable than mPEG-SPA due to its easier synthesis

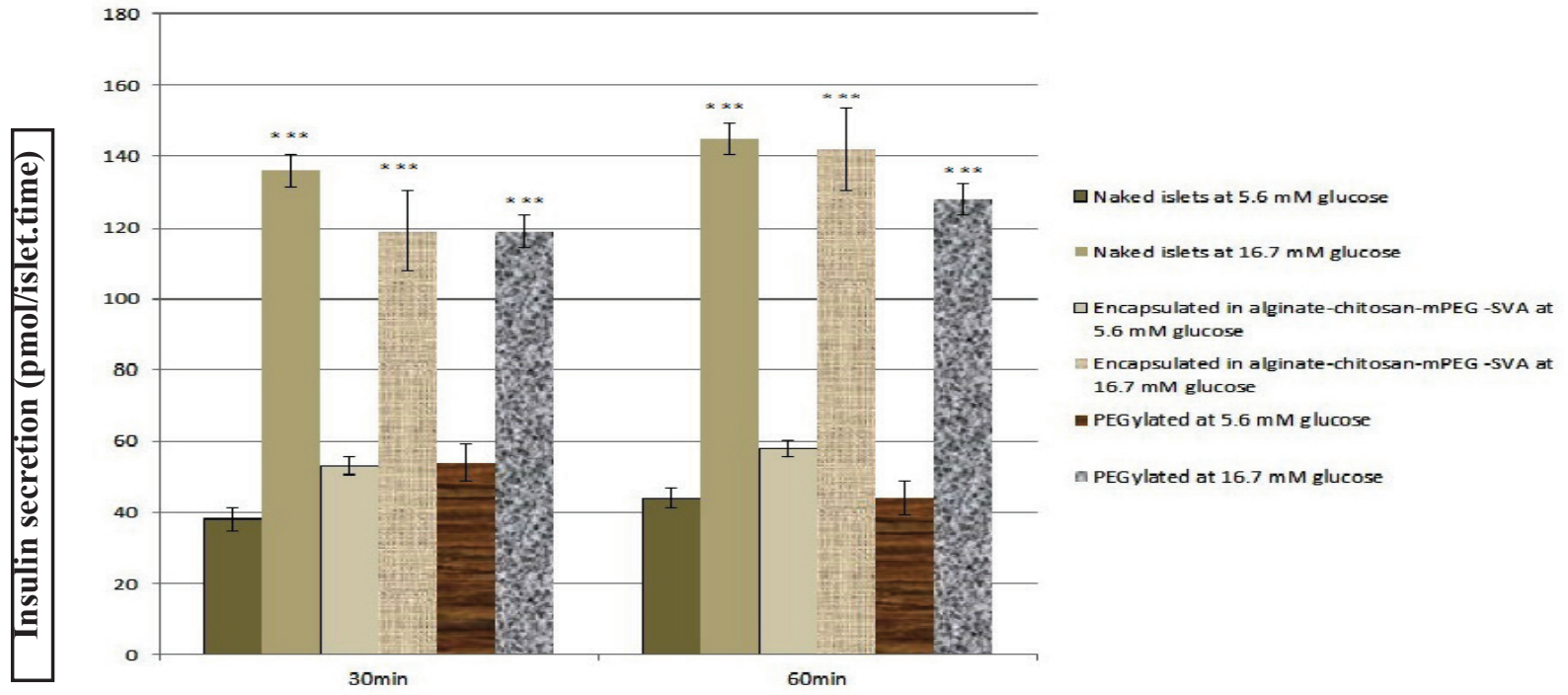

Figure 4. Insulin secretion (pmol/islet.time) from the PEGylated and encapsulated groups compared to the naked islets group in the presence of 5.6 and $16.7 \mathrm{mM}$ glucose concentrations: ( Naked islets at $5.6 \mathrm{mM}$ glucose, naked islets at $16.7 \mathrm{mM}$ glucose, PEGylated islets at 5.6 mM glucose, PEGylated islets at $16.7 \mathrm{mM}$ glucose, encapsulated islets at $5.6 \mathrm{mM}$ glucose, and encapsulated islets at $16.7 \mathrm{mM}$ glucose) (*** shows the significant difference between insulin secretion in the presence of $16.7 \mathrm{mM}$ and $5.6 \mathrm{mM}$ glucose). 
process. Schneider et al. (20) have shown that two layers of the microcapsules using alginate and chitosan can decrease IL-2 secretion and the host's immune stimulation compared to alginate microcapsules. In the previous study (18) We showed $61.9 \%$ decrease in the IL-2 secretion against the encapsulated islets in the alginate-PLO-mPEG-SVA as a novel immunoisolation system using a combination of two different methods of the encapsulation and PEGylation compared to the free islets. The results of the present study show that islets encapsulated in the alginate-chitosan-mPEGSVA as an immunoisolation system, co-cultured with the lymphocytes, significantly decreases IL-2 secretion from lymphocytes; about $38 \%$ less than that co-cultured with the free islets, under the same condition. Also, the viability test showed that the three layer encapsulated islets do not damage the islets as all the islets were alive after encapsulation process.

The immunoisolation systems in addition to protection of the islets against the host immune system should also support their natural functions. This indicates that immunoisolation systems should prepare enough space to transfer glucose, insulin, and other essential nutrients. Korbutt et al (21) induced alginate-encapsulated islets at two different glucose concentrations; at $2.8 \mathrm{mM}$ and $16.7 \mathrm{mM}$, respectively. Their results showed that the alginate capsules had no effect on insulin secretion from the encapsulated islets. Thus, the encapsulated islets can sense glucose as well as control islets. Also, Schneider et al (20) showed that encapsulated islets using alginate-chitosan for encapsulation can release insulin at different glucose concentration; exactly similar to the control islets. Panza et al (2) considered the efficiency of PEG-isocyanate as an immunoisolation system for the islets and reported that the islets in PEG-isocyanate, in addition to stability, can respond to different glucose concentrations. Aghajani-Lazarjani et al (10) exposed the PEGylated islets with mPEG-SPA and mPEG-SC to the different glucose concentrations separately in order to investigate islets function and showed that islets can sensitively respond to the glucose concentrations and release of insulin exactly similar to the free islets. They reported that the difference between insulin secretion from the PEGylated and the free islets was not significant after 60 min incubation.

In the present study, to investigate the performance of islets encapsulated in either alginate-chitosan-mPEG or PEGylated with the mPEG-SVA and in the presence of different glucose concentrations, the amount of insulin secreted from the islets was measured. The PEGylated islets using mPEG-SVA and encapsulated islets in the alginate-chitosan-mPEG exposed to different glucose concentrations could respond to the glucose concentration and release insulin exactly similar to the PEGylated islets with mPEG-SPA and mPEG-SC and retain their biological function. Also, glucose sensitivity for all of the three groups (control, PEGylated with mPEG SVA and encapsulated in alginate-chitosanmPEG SVA) were similar to each other and could release insulin depending on the sensed glucose concentrations (16.7 and $5.6 \mathrm{mM}$ ) after 30 and $60 \mathrm{~min}$. It was shown that three layers of alginate-chitosan-mPEG-SVA have enough space to transfer the essential nutrients as well as insulin. On the other hand, the immune test showed that this kind of immunoisolation system could protect the islets against the host immune system.

\section{Conclusions}

The present study has investigated the effect of encapsulation and PEGylation on the glucose sensitivity of the isolated islets in addition to the insulin release from the encapsulated, PEGylated, and free islets. Insulin release from all of the groups showed that encapsulation and PEGylation have no negative effect on the insulin secretion and glucose sensitivity of the isolated islets. Therefore, these two immunoprotective methods cannot affect the natural function of the islets of Langerhans. Also, it was found that the encapsulated islets in the alginate-chitosan-mPEG-SVA, as an immunoisolation system co-cultured with lymphocytes, have significantly decreased IL-2 secretion from the lymphocytes at about $38 \%$ less than that of co-cultured with the free islets under the same condition.

\section{References}

1. Gaglia JL, James Shapiro AM, Gordon CW. Islet transplantation: Progress and challenge. Arch Med Res. 2005;36(3):273-280. doi: 10.1016/j.arcmed.2005.03.014.

2. Panza JL, Wagner WR, Rilo HL, Rao RH, Beckman EJ, Russell AJ. Treatment of rat pancreatic islets with reactive PEG. Biomaterials. 2000;21(11):1155-1164.

3. Wilson JT, Chaikof EL. Challenges and emerging technologies in the immunoisolation of cells and tissues. Adv Drug Deliv Rev. 2008;60(2):124-145. doi: 10.1016/j.addr.2007.08.034.

4. American Diabetes Association. Diagnosis and classification of diabetes mellitus. Diabetes Care.2004;1:5-10. doi: 10.2337/ dc10-S062.

5. Teramura Y, Iwata H. Bioartificial pancreas: Microencapsulation and conformal coating of islet of Langerhans. Adv Drug Deliv Rev. 2010;62(7):827-840.

6. Beck J, Angus R, Madsen B, Britt D, Vernon B, Nguyen KT. Islet encapsulation: strategies to enhance islet cell functions. Tissue Eng. 2007;13(3):589-599. doi: 10.1089/ten.2006.0183.

7. De Vos P, Faas MM, Strand B, Calafiore R. Alginate-based microcapsules for immunoisolation of pancreatic islets. Biomaterials. 2006;27(32):5603-5617. doi: 10.1016/j. biomaterials.2006.07.010. 
8. Lim F, Sun AM. Microencapsulated islets as bioartificial endocrine pancreas. Science, 1980;210:908-910. doi: 10.1126/ science. 6776628 .

9. De Vos P, Marchetti P. Encapsulation of pancreatic islets for transplantation in diabetes: the untouchable islets. Trends $\mathrm{Mol}$ Med. 2002;8(8):363-366.

10. Aghajani-Lazarjani H, Vasheghani-Farahani E, Shojaosadati SA, Hashemi-Najafabadi S, Zahediasl S, Tiraihi T, et al. The effect of two different polyethylene glycol (PEG) derivatives on the immunological response of PEG grafted pancreatic islets. $J$ Artif Organs. 2010;13(4):218-224. doi : 10.1007/s10047-0100525-9.

11. Aghajani Lazarjani H, Vasheghani-Farahani E, Barani L, Hashemi-Najafabadi S, Shojaosadati SA, Zahediasl S, et al. Effect of polymer concentration on camouflaging of pancreatic islets with mPEG-succinimidyl carbonate. Artif Cells Blood Substit Immobil. 2010;38(5):250-258. doi: 10.3109/10731199.2010.488634.

12. Barani L, Vasheghani-Farahani E, Aghajani-Lazarjani H, Hashemi-Najafabadi S, Atyabi F. Effect of molecular mass of methoxypoly(ethylene glycol) activated with succinimidyl carbonate on camouflaging pancreatic islets. Biotechnol Appl Biochem. 2010;57(1):25-30. doi: 10.1042/BA20100145.

13. Gholami Z, Hashemi-Najafabadi S, Soleimani M. Simultaneous camouflage of major and minor antigens cell surface with activated mPEGs. Iran J Biotechnol. 2014;12(2):15-25.

14. Aghajani-Lazarjani H, Vasheghani-Farahani E, HashemiNajafabadi S, Shojaosadati SA, Zahediasl S, Tiraihi T, et al. Optimization of monomethoxy poly(ethylene glycol) grafting on Langerhans islets capsule using response surface method. Progress in Biomaterials. 2013;2:7. doi: 10.1186\%2F21940517-2-7.
15. Hillberg AL, Oudshoorn M, Lam JB, Kathirgamanathan K. Encapsulation of porcine pancreatic islets within an immunoprotective capsule comprising methacrylated glycol chitosan and alginate. J Biomed Mater Res B Appl Biomater. 2015;103(3):503-518. doi: 10.1002/jbm.b.33185.

16. Azadi SA, Vasheghani-Farahani E, Hashemi-Najafabadi S, Godini A. Co-encapsulation of pancreatic islets and pentoxifylline in alginate-based microcapsules with enhanced immunosuppressive effects. Prog Biomater. 2016;5:101-109. doi: 10.1007/s40204-016-0049-3.

17. Hashemi J, Hashemi-Najafabadi S, Vasheghani-Farahani E. Synergistic effect of PEGylation and pentoxifylline addition on immunoprotection of pancreatic islets. J Biomater Sci. Polymer Edition. 2017;28:33-49. doi: 10.1080/09205063.2016.1239952.

18. Nabavimanesh M, Hashemi-Najafabadi S, Vasheghani-Farahani E. Islets immunoisolation using encapsulation and PEGylation, simultaneously, as a novel design. J Bio Sci Bioeng. 2015; 119(4): 486-491. doi: 10.1016/j.jbiosc.2014.09.023.

19. You S, Rivereau AS, Gouin E, Saï P. Co-incubation of pig islet cells with spleen cells from non-obese diabetic mice causes decreased insulin release by non-T-cell- and T-cell-mediated mechanisms. Clin Exp Immunol. 2001;125(1):25-31. doi: 10.1046/j.1365-2249.2001.01572.x.

20. Schneider S, Feilen PJ, Slotty V, Kampfner D, Preuss S. Multilayer capsules: a promising microencapsulation system for transplantation of pancreatic islets. Biomater. 2001;22(14):19611970. doi: 10.1016/S0142-9612(00)00380-X.

21. Korbutt GS, Mallett AG, Ao Z, Flashner M, Rajotte RV. Improved survival of microencapsulated islets during in vitro culture and enhanced metabolic function following transplantation. Diabetologia, 2004;47(10):1810-1818. doi: 10.1007/s00125-004-1531-3. 